\title{
Peso Adulto de Matrizes em Rebanhos de Seleção da Raça Nelore no Brasil ${ }^{1}$ \\ Antonio do Nascimento Rosa², Raysildo Barbosa Lôbo ${ }^{3}$, Henrique Nunes de Oliveira4, Luiz Antonio Framartino Bezerra ${ }^{5}$, Arcádio de los Reyes Borjas ${ }^{6}$
}

\begin{abstract}
RESUMO - O objetivo deste trabalho foi avaliar a variabilidade fenotípica e genética do peso adulto de matrizes da raça Nelore. As informações trabalhadas foram provenientes de 34 rebanhos, de onze diferentes regiões brasileiras. Foram consideradas na análise vacas das variedades mocha e de chifres, criadas em regime de pasto, de rebanhos com no mínimo 50 matrizes. Com estas restrições, formou-se um conjunto de dados que totalizou 6.867 vacas e uma matriz de parentesco de 17.039 animais. O peso corporal indicador do peso adulto (PA) foi definido como o primeiro peso ( $\mathrm{kg}$ ) observado dentro do limite de quatro a doze anos de idade. Estes pesos foram aferidos em uma das três seguintes condições: normal, nas pesagens de rotina, em abril e outubro de cada ano, de acordo com o calendário estabelecido pelo programa, ao parto ou à desmama do produto. O tratamento de dados e as análises estatísticas foram feitos pela aplicação de procedimentos contidos no Sistema de Análise Estatística (SAS). Fazenda, ano de nascimento, ano de realização da pesagem, situação à pesagem, estação da pesagem e as interações ano*estação de nascimento e ano*estação da pesagem foram as principais causas de variação para PA. A média geral do PA foi $447 \mathrm{~kg}$, com coeficiente de variação de $11 \%$. As análises genéticas foram feitas pela aplicação do modelo animal, utilizando-se o aplicativo MTDFREML, tendo sido formados previamente 1.126 grupos de contemporâneas, reunindo-se as vacas provenientes da mesma fazenda, nascidas no mesmo ano e pesadas no mesmo ano e estação, sob a mesma condição, por ocasião da pesagem. As estimativas dos componentes genético aditivo $\left(537,78 \mathrm{~kg}^{2}\right)$, residual (1.553,22 $\mathrm{kg}^{2}$ ) e fenotípico $\left(2.090,59 \mathrm{~kg}^{2}\right)$, resultaram em uma estimativa de herdabilidade de 0,26 . As predições dos valores genéticos para peso adulto variaram entre $-41,9$ a $+61,4 \mathrm{~kg}$, com acurácias de 0 a 0,95 .
\end{abstract}

Palavras-chave: bovino de corte, maturidade, melhoramento genético, peso corporal, vaca, zebu

\section{Mature Weight of Nellore Cows from Selection Herds in Brazil}

\begin{abstract}
The main objective of this work was to evaluate the Nellore breed in Brazil with respect to the phenotypic and genetic variation of the mature size of cows. The observations were taken from 34 herds of eleven different beef cattle production regions in the country. There were considered cows of the horned and polled varieties, raised on pastures, from herds that had at least 50 cows. With these restrictions it was formed a general file with 6,867 cows with valid observations on mature weight (MW) and a genetic relationship matrix with 17,039 animals. Body weight, as indicator of the mature size, was defined as the first weight (kg) observed since the cows were from four through twelve years old. This weight was taken under one of three different conditions: normal, at the routine weighing, in April and October, according to the established schedule of the program, at parturition or at weaning of the calf. Data management and statistical analyses were carried out through the utilization of the Statistical Analysis System (SAS). Herd, year of birth, year of weighing, condition at weighing, season of weighing and the interactions year*season of birth and year*season of weighing were the most important sources of variation for MW. The overall mean for mature weight was $447 \mathrm{~kg}$, with a coefficient of variation of $11 \%$. The genetic analysis was carried out by the use of the Multiple Trait Derivative-Free Restricted Maximum Likelihood (MTDFREML) algorithm under an animal model. The 1,126 contemporary groups included cows from the same herd, born at the same year, weighted at the same year and season and the same condition at weighing. The additive genetic $\left(537.78 \mathrm{~kg}^{2}\right)$, residual $\left(1,553.22 \mathrm{~kg}^{2}\right)$ and phenotypic $\left(2,090.59 \mathrm{~kg}^{2}\right)$ variance components provided a heritability estimate of 0.26 , for MW. The predicted genetic breeding values varied from -41.9 through $+61.4 \mathrm{~kg}$, with accuracy ranging from 0 to 0.95 .
\end{abstract}

Key Words: animal breeding, beef cattle, body weight, cow, maturity, zebu cattle

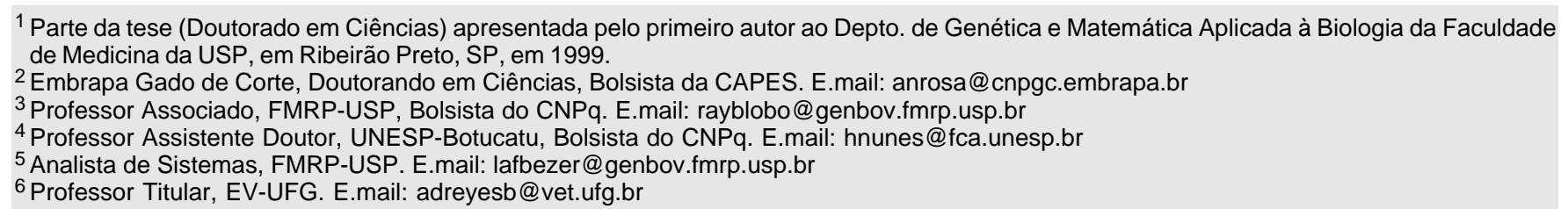




\section{Introdução}

O peso adulto das matrizes vem despertando atenção e discussões, nos últimos anos, em função da globalização da economia que exige, a cada dia, além da qualidade, baixos custos de produção, como forma de se enfrentar um mercado de alta competitividade (ANUALPEC, 1998; HOHENBOKEN, 1998; LANNA e PACKER, 1998).

A razão fundamental é que o tamanho, do qual o peso corporal é um dos melhores indicadores, afeta os custos de mantença e a eficiência biológica e econômica dos rebanhos (FITZHUGH, 1976). Além disto, é relacionado à estrutura corporal, harmonia e equilíbrio dos animais que, juntamente com outras características fisiológicas, atuam diretamente sobre os mecanismos de adaptabilidade ao meio ambiente (PETERS, 1993).

Quando a criação é feita em condições pouco modificadas, em regime de pastagens, por exemplo, o meio ambiente determina os limites até os quais as modificações genéticas poderão ter sucesso. No entanto, em sistemas de criação intensiva, com práticas sofisticadas de manejo alimentar, reprodutivo e sanitário, podem ser grandes os perigos de se extrapolar os limites que a biologia do animal poderá suportar, com equilíbrio, comprometendo-se o bem estar, o conforto e, por conseqüência, os níveis de produção, reprodução e longevidade dos animais (HOHENBOKEN, 1996).

O consenso da literatura a respeito deste tema é que animais de grande porte podem ser mais eficientes em ambientes com fartura de alimentos. Em outros, com restrições, principalmente nutricionais, devem ser preferidos os de porte médio, ou até pequeno (JENKINS e FERREL, 1994; RITCHIE, 1995).

De modo geral, o Brasil pode ser considerado um país privilegiado, em função da variabilidade de ambientes disponíveis (ARRUDA e SUGAI, 1994), que possibilita a exploração de uma grande diversidade de recursos genéticos. Considerando-se as regiões Sudeste, Centro-Oeste, Nordeste, Amazônia Oriental e Ocidental, onde predominam, na pecuária de corte, as raças de origem indiana, são verificados diferentes tipos de clima e de solos, com a ocorrência de terras de cultura, cerradão, cerrados, campos limpos, matas equatoriais, caatinga e pantanal, que abrem espaço para a criação de vários biótipos, em diferentes sistemas de produção.

Muito progresso poderá ser alcançado na eficiência destes sistemas, aliando-se a disponibilidade des- tas condições naturais, favoráveis a uma exploração comercial ecologicamente adequada e com custos de produção compatíveis, a um maior conhecimento das potencialidades dos recursos genéticos. A este respeito, no caso da raça Nelore, que predomina nos sistemas de produção do Brasil Central, além de raros, a maioria dos trabalhos relativos ao tamanho adulto foi realizada como resultados do ajuste de curvas exponenciais ao crescimento (CORTARELLI, 1973; DUARTE, 1975; LUDWIG, 1979; ROSA et al., 1978; NOBRE et al., 1987b; ELIAS et al., 1998), com problemas de amostragem, em relação à raça como um todo, ou com pares de observações "pesoidade" que, não envolvendo satisfatoriamente todo o ciclo de vida dos animais, não permitem um ajuste adequado dos modelos de crescimento.

ROSA et al. (2000) iniciaram um estudo desta natureza, a partir de dados de campo, tendo analisado, no entanto, as informações de um único rebanho no Estado de São Paulo.

Nesta oportunidade, após a atualização da base de dados do Programa de Melhoramento Genético da Raça Nelore (LÔBO, 1996), o objetivo é apresentar uma avaliação mais ampla, quer em termos de população quer em termos de ambiente, de modo a se fornecer subsídios que proporcionem a adequação dos genótipos, pela aplicação de seleção, às diferentes condições ambientais, visando o aumento da produtividade da raça.

\section{Material e Métodos}

Foram analisadas as informações de 34 rebanhos, provenientes de onze diferentes regiões de produção (RP) de gado de corte do Brasil (Tabela 1), integrantes do Programa de Melhoramento Genético da Raça Nelore, em execução desde 1988 (LÔBO, 1996). As regiões de produção de gado de corte no Brasil foram propostas por ARRUDA e SUGAI (1994), a partir de análises conjuntas dos sistemas de produção e das condições de solo, clima e vegetação predominantes nas microregiões homogêneas, conforme classificação original do Instituto Brasileiro de Geografia e Estatística.

Com exceção da região 10, do Araguaia, localizada na Amazônia Oriental, com clima Equatorial, todas as demais regiões estão incluídas nas regiões CentroOeste e Sudeste, onde o clima varia de Subtropical Quente Úmido, com temperaturas médias anuais mais amenas $\left(18-20^{\circ} \mathrm{C}\right)$ ao sul de Mato Grosso do Sul, a Tropical Quente Úmido, na região de Itapetinga, 
Rev. bras. zootec.

Tabela 1 - Relação das fazendas por código, nome, município, estado, região de produção de gado de corte e ano de início no programa de melhoramento

Table 1 - Name and code of the ranches, county, state, beef cattle production region and year at the beginning of the animal breeding program

\begin{tabular}{|c|c|c|c|c|}
\hline $\begin{array}{l}\text { Código } \\
\text { Code }\end{array}$ & $\begin{array}{l}\text { Nome } \\
\text { Name }\end{array}$ & $\begin{array}{l}\text { Município, Estado } \\
\text { County, State }\end{array}$ & $\begin{array}{l}\text { Região: Código e Nome }{ }^{1} \\
\text { Region: Code and Name }{ }^{1}\end{array}$ & $\begin{array}{l}\text { Ano } \\
\text { Year }\end{array}$ \\
\hline 01 & Santa Marta & Naviraí, MS & 18 C. Grande-Dourados & 1988 \\
\hline 02 & Chácara Naviraí & Uberaba, MG & 31 Triângulo Mineiro & 1988 \\
\hline 03 & Europa & Uberaba, MG & 31 Triângulo Mineiro & 1988 \\
\hline 05 & Ivaé & Ivinhema, MS & 18 C. Grande-Dourados & 1988 \\
\hline 06 & Guanacaste & Uberaba, MG & 31 Triângulo Mineiro & 1990 \\
\hline 08 & M. Vermelho & Jaú, SP & 38 Araraquara & 1991 \\
\hline 09 & S. Â. Guirahy & Jateí, MS & 18 C. Grande-Dourados & 1991 \\
\hline 10 & Eldorado & Santa Inês, MA & 10 Araguaia & 1992 \\
\hline 12 & Terra Boa & Guararapes, SP & 37 Oeste de São Paulo & 1992 \\
\hline 13 & Mate Amargo & Rio Brilhante, MS & 18 C. Grande-Dourados & 1993 \\
\hline 14 & Cambira & Itaúna, MG & 39 Região Leiteira & 1993 \\
\hline 16 & Santa Filomena & Ocauçú, SP & 37 Oeste de São Paulo & 1994 \\
\hline 17 & Baguaçu & Uberaba, MG & 31 Triângulo Mineiro & 1994 \\
\hline 18 & Brumado POI & Barretos, SP & 38 Araraquara & 1994 \\
\hline 19 & Renascença & Unaí, MG & 32 Oeste Mineiro & 1994 \\
\hline 20 & São Luiz & Barra do Garça, MT & 15 Rondonópolis & 1994 \\
\hline 21 & Boa Vista & Anhembi, SP & 38 Araraquara & 1994 \\
\hline 22 & Rancho Verde & Caarapó, MS & 18 C. Grande-Dourados & 1994 \\
\hline 24 & Boa Esperança & Uberaba, MG & 31 Triângulo Mineiro & 1994 \\
\hline 26 & S. L. Coqueiros & Jaborandi, SP & 38 Araraquara & 1995 \\
\hline 28 & Divisão & Amambaí, MS & 18 C. Grande-Dourados & 1995 \\
\hline 29 & Cambará & Sidrolândia, MS & 18 C. Grande-Dourados & 1995 \\
\hline 30 & Boticão & Barretos, SP & 38 Araraquara & 1995 \\
\hline 31 & Aricá & Aruanã, GO & 20 Alto Tocantins & 1995 \\
\hline 33 & Rancho Alegre & Campo Grande, MS & 18 C. Grande-Dourados & 1996 \\
\hline 34 & Brumado PO & Barretos, SP & 38 Araraquara & 1997 \\
\hline 35 & Eldorado & Lajedão, BA & 35 Itapetinga-Valadares & 1997 \\
\hline 36 & Cinelândia & Lajedão, BA & 35 Itapetinga-Valadares & 1997 \\
\hline 39 & Lagoa do Buriti & Uberaba, MG & 31 Triângulo Mineiro & 1993 \\
\hline 50 & São Dimas & S. M. Araguaia, GO & 20 Alto Tocantins & 1995 \\
\hline 51 & Cabaçal & Veríssimo, MG & 31 Triângulo Mineiro & 1995 \\
\hline 52 & Campina & Caiuá, SP & 37 Oeste de São Paulo & 1994 \\
\hline 53 & Salto & Itaguaçu, GO & 30 Goiás & 1995 \\
\hline 54 & Prov. V. Verde & S. M. Araguaia, GO & 20 Alto Tocantins & 1995 \\
\hline
\end{tabular}

${ }^{1}$ Fonte: ARRUDA e SUGAI (1994).

Bahia, com temperaturas um pouco mais elevadas (Tabela 2). Em geral, a média anual da precipitação pluviométrica nestas regiões varia de 1.100 , no Alto Tocantins (RP 20), a 1.900 mm, na região do Araguaia. Geralmente, o período seco do ano situa-se entre maio e setembro, com média de duração variando de dois (RP 39) a cinco meses (RP 32) (ARRUDA e SUGAI, 1994).

O regime pluviométrico e os diferentes tipos de solo disponíveis certamente são as principais causas de variação entre regiões e entre fazendas, uma vez que determinam a quantidade e a qualidade das pastagens que podem ser ofertadas aos rebanhos. Geralmente, nas regiões com solos de baixa a média fertilidade são cultivadas gramíneas do gênero Brachiaria, principalmente das espécies Brachiaria decumbens e Brachiaria humidicola.

Todas as fazendas participantes do programa adotam estação de monta, com duração média de 60 a 90 dias, realizada no período entre outubro e março. O início desta estação pode variar entre regiões ou período de tempo, em função das condições climáticas disponíveis a cada ano. Predomina entre os criadores a prática da inseminação artificial com repasse de touros, em caso de retorno ao cio, após a primeira ou segunda inseminação. A desmama dos bezerros é realizada geralmente com a idade média de sete a oito meses e os cuidados sanitários e de vacinações são feitos conforme estabelecido pelos órgãos de controle sanitário de cada região.

A extração dos dados da base geral foi feita selecionando-se rebanhos com, no mínimo, 50 matrizes das variedades mocha e de chifres, nascidas entre 1982 e 1993, criadas em condições de pastagens, cujas pesagens foram tomadas entre quatro e 
ROSA et al.

Tabela 2 - Fertilidade de solo, clima e vegetação original predominantes nas regiões de produção (RP) de gado de corte envolvidas nas análises ${ }^{1}$

Table 2 - Soil fertility, climate and original predominant vegetation on the beef cattle production regions (RP) involved in the analyses

\begin{tabular}{llll}
\hline RP & $\begin{array}{c}\text { Solo: fertilidade, classificação } \\
\text { Soil: fertility, } \text { classification }^{2}\end{array}$ & \multicolumn{1}{c}{$\begin{array}{c}\text { Clima } \\
\text { Climate }\end{array}$} & \multicolumn{1}{c}{$\begin{array}{c}\text { Vegetação original } \\
\text { Original vegetation }\end{array}$} \\
\hline 10 & Média,LVA/LVE & Quente Equatorial Úmido & Mata Equatorial/Cerrado \\
15 & Baixa, AQ & Quente Semi-úmido & Cerrado \\
18 & Média-Alta, LVE/R & Subtropical Quente Úmido & Mata/Cerrado/Campo \\
20 & Média, LVA & Quente Semiúmido & Mata Tropical \\
30 & Média-Alta, LVA/VE & Subtropical Quente Úmido & Mata Tropical/Cerrado \\
31 & Média-Alta, LVE/R & Tropical Úmido/Semiúmido & Mata/Cerrado \\
32 & Baixa-Média, LVA/AQ & Tropical Quente Semiúmido & Cerrado/Caatinga \\
35 & Alta,LVE & Tropical Quente Úmido & Mata Tropical \\
37 & Média-Alta, LR/VE & Tropical Quente Úmido & Mata Tropical \\
38 & Média-Alta, LR/VA/AQ & Tropical Úmido/Superúmido & Mata Tropical/Cerrado \\
39 & Média-alta, LVE & Tropical Subúmido-Úmido & Mata Tropical/Cerrado \\
\hline
\end{tabular}

1 ARRUDA E SUGAI (1994).

2 LVA, LVE, LR, AQ = latossolo vermelho amarelo (red yellow latosoil), latossolo vermelho escuro (dark red latosoil), latossolo roxo (purple latosoil), e areia quartzosa (quartz sand), respectivamente (respectively).

doze anos de idade.

O tratamento dos dados e as análises estatísticas para a identificação de fontes de variação de meio ambiente e para a formação dos grupos de contemporâneas, como etapa inicial para as análises genéticas, foram feitas pela aplicação dos procedimentos disponíveis no Sistema de Análises Estatísticas (SAS, 1995). Nesta primeira etapa, o modelo fixo geral aplicado pode ser descrito da seguinte forma:

$$
\mathrm{y}=\mathrm{X} \beta+\mathrm{e}
$$

em que: $\mathrm{y}=$ vetor das observações; $\mathrm{X}=$ matriz de incidência dos efeitos fixos devido à região de produção, fazenda, ano e estação de nascimento, ano, estação e situação à pesagem; $\beta=$ vetor dos efeitos fixos desconhecidos; $\mathrm{e}=$ vetor dos efeitos residuais.

$\mathrm{Na}$ aplicação deste modelo, as pressuposições assumidas foram: $\mathrm{E}(\mathrm{y})=\mathrm{X} \beta ; \mathrm{E}(\mathrm{e})=0$ e $\operatorname{Var}(\mathrm{y})=\operatorname{Var}(\mathrm{e})=$ $I_{N} \sigma_{e}^{2}$, sendo I uma matriz identidade quadrada de ordem $\mathrm{N}$, onde $\mathrm{N}$ é o número de vacas com observações.

Para a estimação dos componentes de variância e dos parâmetros genéticos, foi aplicado o procedimento de máxima verossimilhança restrita, sob modelo animal, disponível no aplicativo MTDFREML (BOLDMAN et al., 1993).

Neste caso, o modelo linear misto foi definido da seguinte forma:

$$
y=\mathrm{X} \beta+\mathrm{Za}+e
$$

em que: $\mathrm{y}=$ vetor das observações; $\mathrm{X}=$ matriz de incidência dos efeitos fixos, devidos aos grupos de contemporâneas: fazenda-ano de nascimento-ano e estação de realização das pesagens-condição à pesagem; $\beta=$ vetor dos efeitos fixos desconhecidos relati- vos aos grupos de contemporâneas; $\mathrm{Z}=$ matriz quadra$\mathrm{da}$, de ordem $\mathrm{N}$, que relaciona as observações de y com os respectivos efeitos genéticos aditivos dos animais, contidos em a; $\mathrm{a}=$ vetor dos efeitos genéticos aditivos a serem preditos; $\mathrm{e}=$ vetor dos efeitos residuais.

As pressuposições assumidas para a aplicação deste modelo foram: $\mathrm{E}[\mathrm{a}]=\mathrm{E}[\mathrm{e}]=0$ e $\mathrm{E}[\mathrm{y} \mid \mathrm{a}]=\mathrm{X} \beta$. A distribuição conjunta dos efeitos aleatórios pode ser descrita pela seguinte relação:

$$
\operatorname{Var}\left[\begin{array}{l}
a \\
e
\end{array}\right]=\left[\begin{array}{cc}
A \sigma_{a}^{2} & 0 \\
0 & I_{N} \sigma_{e}^{2}
\end{array}\right]
$$

em que: $\sigma_{a}^{2}=$ variância genética aditiva direta; $\mathrm{A}=$ matriz dos coeficientes de parentesco, de Wright, entre os animais; $\sigma_{e}^{2}=$ variância residual; $\mathrm{I}=$ matriz identidade de ordem $\mathrm{N} ; \mathrm{N}=$ número de matrizes com observações.

A herdabilidade do peso adulto, disponível na saída do programa MTDFREML, é obtida pela expressão:

$$
h^{2}=\sigma_{a}^{2} /\left(\sigma_{a}^{2}+\sigma_{e}^{2}\right)
$$

\section{Resultados e Discussão}

As principais regiões de produção em termos de número de fazendas e de animais, quais sejam, Campo Grande-Dourados (RP 18), Alto Tocantins (RP 20), Triângulo Mineiro (RP 31), Oeste de São Paulo (RP 37) e Araraquara (RP 38), envolvendo 27 das 34 fazendas (Tabela 1), foram reunidas em um primeiro arquivo de trabalho, com o objetivo de se analisar a variabilidade do peso adulto das matrizes, em função das diferentes regiões de produção. 
Rev. bras. zootec.

Ao se aplicar um modelo linear com a fonte de variação fazenda aninhada em região, verificaramse efeitos significativos de variedade (Nelore padrão ou mocho), tipo de pesagem (de rotina, ao parto ou à desmama), ano de nascimento, ano, estação e interação entre ano e estação da pesagem ( $\mathrm{P}<0,0012$; Tabela 3$)$.

A região de Araraquara apresentou estimativa mais elevada de peso adulto (460 kg), vindo em seguida a região de Campo Grande-Dourados (445 kg), Triângulo Mineiro e Oeste de São Paulo (432 e $431 \mathrm{~kg}$ ) e Alto Tocantins (387 kg, Tabela 4). Desta forma, os maiores valores para peso adulto foram verificados nas regiões com os melhores recursos de ambiente disponíveis (Tabela 2), como também observou SILVA (1990), embora trabalhando com animais jovens.

Com relação aos efeitos de região e de fazenda, no entanto, observou-se maior contribuição do efeito de fazenda aninhado em região do que do efeito de região, como efeito principal, embora ambos significativos ( $\mathrm{P}<0,0001$; Tabela 3$)$. Resultados semelhantes a estes foram relatados por NOBRE et al. (1987a) e SILVA (1990), entre outros autores. Provavelmente, embora os elementos de clima, principalmente, sejam bastante homogêneos dentro de cada região (ARRUDA e SUGAI, 1994), outros fatores tais como tipos de solos, pastagens e manejo dos animais, além do seu próprio valor genético, podem estar contribuindo para acentuar as diferenças entre rebanhos.

Desta forma, tendo-se em vista uma abordagem a mais ampla possível com relação à amostra disponível, os dados referentes a todas as 34 fazendas foram submetidos à análise de variância por um modelo linear geral de classificação cruzada.

Neste caso, fazenda, ano de nascimento e ano de realização da pesagem foram as principais fontes de variação para peso adulto, embora tenham sido também significativos os efeitos de situação à pesagem, estação da pesagem e das interações ano*estação de nascimen-

Tabela 3 - Análise de variância do peso adulto por um modelo fixo incluindo o efeito da região de produção e fazenda dentro de região

Table 3 - General linear model procedure for mature weight by a fixed model including the beef cattle production region and herd within region effects

\begin{tabular}{|c|c|c|c|c|c|}
\hline $\begin{array}{l}\text { Fonte de variação } \\
\text { Source of variation }\end{array}$ & $\begin{array}{l}\text { G } \\
D F\end{array}$ & $\begin{array}{l}\text { Soma de quadrados } \\
\text { Sum of squares }\end{array}$ & $\begin{array}{l}\text { Quadrado médio } \\
\text { Means square }\end{array}$ & $\mathrm{F}$ & $\mathrm{P}>\mathrm{F}$ \\
\hline $\begin{array}{l}\text { Região }(\mathrm{R}) \\
\operatorname{Region}(R)\end{array}$ & 4 & 1.358 .470 & 339.617 & 137,0 & 0,0001 \\
\hline $\begin{array}{l}\text { Fazenda:R } \\
\text { Herd:R }\end{array}$ & 22 & 4.763 .773 & 216.535 & 87,3 & 0,0001 \\
\hline $\begin{array}{l}\text { Variedade }^{1} \\
\text { Variety }^{1}\end{array}$ & 1 & 49.325 & 49.325 & 19,9 & 0,0001 \\
\hline $\begin{array}{l}\text { Tipo de pesagem }{ }^{2} \\
\text { Kind of weighing }^{2}\end{array}$ & 2 & 150.096 & 75.048 & 30,3 & 0,0001 \\
\hline $\begin{array}{l}\text { Ano }(\mathrm{A})^{3} \\
\text { Year }(Y)^{3}\end{array}$ & 11 & 778.128 & 70.739 & 28,5 & 0,0001 \\
\hline $\begin{array}{l}\text { Estação }(\mathrm{E})^{3} \\
\text { Season }(S)^{3}\end{array}$ & 1 & 274 & 274 & 0,1 & 0,7396 \\
\hline $\begin{array}{l}\mathrm{A} * \mathrm{E} \\
Y^{*} \mathrm{~S}\end{array}$ & 11 & 42.905 & 3900 & 1,6 & 0,0995 \\
\hline $\begin{array}{l}\mathrm{AP}^{4} \\
Y W^{4}\end{array}$ & 9 & 573.862 & 63.762 & 25,7 & 0,0001 \\
\hline $\begin{array}{l}\mathrm{EP}^{4} \\
S W^{4}\end{array}$ & 1 & 26.026 & 26.026 & 10,5 & 0,0012 \\
\hline $\begin{array}{l}\text { AP*EP } \\
Y W^{*} S W\end{array}$ & 9 & 76.859 & 8540 & 3,4 & 0,0003 \\
\hline $\begin{array}{l}\text { Modelo } \\
\text { Model }\end{array}$ & 71 & 12.697 .093 & 178.832 & 72,1 & 0,0001 \\
\hline $\begin{array}{l}\text { Resíduo } \\
\text { Error }\end{array}$ & 5331 & 13.217 .059 & 2479 & & \\
\hline $\begin{array}{l}\text { Total corrigido } \\
\text { Corrected total }\end{array}$ & 5402 & 25.914 .152 & & & \\
\hline
\end{tabular}

${ }_{1}^{1}$ Mocha e de chifres (Polled or horned).

2 De rotina (abril e outubro), ao parto ou à desmama (routine weighing, at parturition or at weaning).

${ }^{3} \mathrm{~A}=$ ano de nascimento; $\mathrm{E}=$ estação de nascimento $(Y=$ year of birth; $S=$ season of birth).

${ }^{4} \mathrm{AP}=$ ano da pesagem; $\mathrm{EP}=$ estação da pesagem ( $Y W=$ year of weighing; $S W=$ season of weighing). 
1032

Tabela 4 - Número de observações (N) e médias estimadas por quadrados mínimos para peso adulto, por região de produção

Table 4 - Number of observations $(N)$ and mature weight least squares means by beef cattle production region

\begin{tabular}{lll}
\hline Região de produção & N Médias $(\mathrm{kg})$
\end{tabular}

Production region Means $(\mathrm{kg})$

\begin{tabular}{lll}
\hline Araraquara-38 & 738 & 460
\end{tabular}

Campo Grande-Dourados-18 $1539 \quad 445$

Triângulo Mineiro-31 $\quad 1126 \quad 432$

Oeste de São Paulo-37 $\quad 1297 \quad 431$

Alto Tocantins-20

$703 \quad 387$

to e ano*estação da pesagem ( $\mathrm{P}<0,0001$; Tabela 5).

A média geral estimada por quadrados mínimos para peso adulto foi $447 \mathrm{~kg}$, sendo o coeficiente de variação igual a $11 \%$, correspondente a um desvio médio do erro de $50 \mathrm{~kg}$. Desta forma, o peso adulto entre 400 e $500 \mathrm{~kg}$, caracteriza a raça Nelore como uma raça de porte médio, conforme classificação das raças bovinas apresentada por ENSMINGER (1987).

O valor médio aqui encontrado coincide com o peso adulto reportado por FELIUS (1985), $445 \mathrm{~kg}$, a partir de ampla revisão de literatura de dados de campo, provenientes da raça Nelore de várias partes do mundo.

Os efeitos relativos à fazenda podem estar incluindo uma série muito complexa de fatores locais, como tipos de solo, pastagens, manejos nutricional, sanitário e reprodutivo e valor genético dos animais, bem como também regionais, em termos principalmente de fatores climáticos (NOBRE et al., 1987a; SILVA, 1990).

Com relação ao ano de realização das pesagens, observou-se tendência de aumento do peso adulto no decorrer do período, provavelmente como reflexo de diferenças ambientais e da tendência de aumento do número de animais, principalmente a partir de 1992, com o ingresso de novos rebanhos no programa (Tabela 1, Tabela 6).

As médias de quadrados mínimos para pesagens realizadas na estação de novembro-abril (450 $\mathrm{kg}$ ) foram semelhantes às realizadas em maiooutubro $(433 \mathrm{~kg})$. Por outro lado, pesos tomados em abril ou outubro, de acordo com o calendário do programa $(448 \mathrm{~kg})$, ou ao parto $(447 \mathrm{~kg})$ foram superiores aos registrados por ocasião da desmama dos produtos (429 kg; Tabela 6), refletindo os desgastes naturais da amamentação e as carências alimentares às quais estão sujeitas as vacas, durante este período.
OSA et al.

Tabela 5 - Resumo da análise de variância do peso adulto Table 5 - General linear model procedure for mature weight

\begin{tabular}{|c|c|c|c|c|}
\hline $\begin{array}{l}\text { Fonte de } \\
\text { variação } \\
\text { Source of } \\
\text { variation }\end{array}$ & $\begin{array}{l}\mathrm{G} \\
D F\end{array}$ & $\begin{array}{l}\text { Quadrado } \\
\text { médio } \\
\text { Mean } \\
\text { square }\end{array}$ & $\mathrm{F}$ & $\operatorname{Pr}>\mathrm{F}$ \\
\hline $\begin{array}{l}\text { Fazenda } \\
\text { Herd }\end{array}$ & 33 & $273.290,31$ & 108,53 & 0,0001 \\
\hline $\begin{array}{l}\text { Tipo de pesagem } \\
\text { Kind of weighing } 1\end{array}$ & 2 & $93.353,67$ & 37,07 & 0,0001 \\
\hline $\begin{array}{l}\text { Ano }(\mathrm{A})^{2} \\
\operatorname{Year}(Y)^{2}\end{array}$ & 11 & $94.912,46$ & 37,69 & 0,0001 \\
\hline $\begin{array}{l}\text { Estação }(\mathrm{E})^{2} \\
\text { Season }(S)^{2}\end{array}$ & 1 & 426,96 & 0,17 & 0,6805 \\
\hline $\begin{array}{l}\mathrm{A}^{* \mathrm{E}} \\
Y * S\end{array}$ & 11 & $5.470,0$ & 2,17 & 0,0133 \\
\hline $\begin{array}{l}\mathrm{AP}^{3} \\
Y W^{3}\end{array}$ & 9 & $90.740,65$ & 36,04 & 0,0001 \\
\hline $\begin{array}{l}\mathrm{EP}^{3} \\
S W^{3}\end{array}$ & 1 & $83.793,70$ & 33,28 & 0,0001 \\
\hline $\begin{array}{l}\mathrm{AP} * \mathrm{EP} \\
Y W * S W\end{array}$ & 9 & $10.979,06$ & 4,36 & 0,0001 \\
\hline $\begin{array}{l}\text { Modelo } \\
\text { Model }\end{array}$ & 77 & $207.130,13$ & 82,26 & 0,0001 \\
\hline $\begin{array}{l}\text { Resíduo } \\
\text { Error }\end{array}$ & 6789 & $2.518,06$ & & \\
\hline $\begin{array}{l}\text { Total corrigido } \\
\text { Corrected total }\end{array}$ & 6866 & & & \\
\hline $\mathrm{R}^{2}$ & $\mathrm{CV}(\%)$ & & $\begin{array}{l}\text { lédia }(\mathrm{kg}) \\
\text { Mean }(\mathrm{kg})\end{array}$ & \\
\hline 0,48 & 11 & & 447 & \\
\hline
\end{tabular}

1 De rotina (abril e outubro), ao parto ou à desmama (routine weighing, at parturition or at weaning).

${ }^{2} \mathrm{~A}=$ ano de nascimento; $\mathrm{E}$ = estação de nascimento $(Y=$ year of birth; $S=$ season of birth).

${ }^{3} \mathrm{AP}=$ ano da pesagem; $\mathrm{EP}=$ estação da pesagem $(Y W=$ year of weighing; $S W=$ season of weighing).

As médias estimadas por quadrados mínimos para peso adulto, por ano e estação de nascimento das vacas, são apresentadas na Tabela 7. Em geral, as médias dos pesos de vacas nascidas na estação de maio a outubro foram inferiores às de outras, nascidas entre novembro e abril, com exceção dos anos 1985 e 1989 que apresentaram comportamento inverso.

As médias foram decrescentes no período analisado, 1982 a 1993, para qualquer das estações. Diferenças devidas ao ano de nascimento das vacas refletem todas as alterações fenotípicas totais ocorridas no período. Estas podem ser de origem natural, como aquelas provocadas por alterações climáticas, por exemplo, ou antrópicas, ou seja provocadas pela ação do criador, como no caso de práticas de manejo alimentar, reprodutivo e sanitário dos rebanhos. A estas forças são também acrescidas as modificações 
Rev. bras. zootec.

Tabela 6 - Número de vacas $(\mathrm{N})$ e médias de quadrados mínimos do peso adulto por ano, estação e tipo de pesagem

Table 6 - Number of cows $(N)$ and least squares means for mature weight by year, season and kind of weighing

\begin{tabular}{lcc}
\hline $\begin{array}{c}\text { Ano } \\
\text { Year }\end{array}$ & $\mathrm{N}$ & $\begin{array}{c}\text { Média }(\mathrm{kg}) \\
\text { Mean }(\mathrm{kg})\end{array}$ \\
\hline 1988 & 60 & 365 \\
1989 & 99 & 397 \\
1990 & 100 & 413 \\
1991 & 122 & 427 \\
1992 & 581 & 444 \\
1993 & 1.407 & 442 \\
1994 & 1.541 & 460 \\
1995 & 1.288 & 474 \\
1996 & 944 & 487 \\
1997 & 725 & 506 \\
\hline
\end{tabular}

Estação

Season

\begin{tabular}{lcc}
\hline $\begin{array}{l}\text { Maio-outubro } \\
\text { May-October }\end{array}$ & 3969 & 433 \\
$\begin{array}{l}\text { Novembro-abril } \\
\text { November-April }\end{array}$ & 2898 & 450 \\
\hline $\begin{array}{l}\text { Tipo de pesagem } \\
\text { Kind of weighing }\end{array}$ & & \\
\hline $\begin{array}{l}\text { De rotina } \\
\text { Routineweighing }\end{array}$ & 5072 & 448 \\
$\begin{array}{l}\text { À desmama } \\
\text { Atweaning } \\
\text { Ao parto } \\
\text { At parturition }\end{array}$ & 698 & 429 \\
\hline
\end{tabular}

${ }^{1} \mathrm{Em}$ abril ou outubro, de acordo com o calendário do programa.

1 In April or October, according to the program schedule.

genéticas dos animais como respostas aos métodos de seleção, como também observaram NOBRE et al. (1987a) e SILVA (1990).

A tendência de decréscimo nas médias de quadrados mínimos, no decorrer do período, poderia ser creditada, em princípio, a mudanças ambientais, especialmente com relação à degradação das pastagens que vem ocorrendo nas áreas de cerrado do Brasil desde o final da década de 80 (ANUALPEC, 1998). No entanto, muitos outros fatores devem estar contribuindo para esta variação. Ao longo do período de execução do programa, foram sendo incorporados novos rebanhos com valores fenotípicos inferiores aos rebanhos incluídos no programa desde o início, em 1988. Outro aspecto a ser considerado é que além do descarte de vacas de grande porte, que pode ter ocorrido em função de esclarecimentos junto aos criadores participantes do programa ou, simplesmente, por terem apresentado baixos índices de eficiência reprodutiva, vem se registrando, no decorrer deste período, uma redução da idade de ingresso de novilhas em reprodução (LÔBO, 1996) que pode limitar a expressão fenotípica máxima do peso adulto.

Para a realização das análises genéticas, pela aplicação do modelo animal, foram formados, previamente, 1126 grupos de contemporâneas reunindo-se em cada grupo as vacas com dados válidos de peso adulto, provenientes da mesma fazenda, nascidas no mesmo ano e pesadas no mesmo ano e estação, sob a mesma condição, à pesagem. Após as restrições impostas com relação ao número mínimo de dois animais, restaram 758 grupos, cuja média e desvio-padrão do número de animais foram, respectivamente, 8,6 e 12 animais, sendo o máximo observado de 164 .

Os parâmetros iniciais utilizados na estimação dos componentes de variância para peso adulto foram aqueles estimados previamente para um rebanho Nelore do Estado de São Paulo (ROSA et al., 2000), ou seja $561 \mathrm{~kg}^{2}$ para variância genética aditiva e $983 \mathrm{~kg}^{2}$, para variância residual.

Com a convergência do processo de máxima verossimilhança, após reinício do programa, para garantir-se o máximo global com critério de convergência igual a $10^{-9}$, foram obtidas as estimativas dos componentes genético aditivo $\left(537,78 \mathrm{~kg}^{2}\right)$, residual

Tabela 7 - Número de vacas $(\mathrm{N})$ e médias de quadrados mínimos do peso adulto por ano, estação e tipo de pesagem

Table 7 - Number of cows $(N)$ and least squares means for mature weight by year and season of birth

\begin{tabular}{|c|c|c|c|c|}
\hline \multirow[t]{4}{*}{$\begin{array}{l}\text { Ano } \\
\text { Year }\end{array}$} & \multicolumn{4}{|c|}{$\begin{array}{l}\text { Estação de nascimento } \\
\text { Season of birth }\end{array}$} \\
\hline & \multicolumn{2}{|c|}{$\begin{array}{l}\text { Maio-outubro } \\
\text { May-October }\end{array}$} & \multicolumn{2}{|c|}{$\begin{array}{l}\text { Novembro-abril } \\
\text { November-April }\end{array}$} \\
\hline & $\mathrm{N}$ & Médias (kg) & $\mathrm{N}$ & Médias (kg) \\
\hline & $N$ & Means (kg) & $N$ & Means (kg) \\
\hline 1982 & 70 & 483 & 38 & 490 \\
\hline 1983 & 170 & 468 & 105 & 475 \\
\hline 1984 & 225 & 463 & 143 & 470 \\
\hline 1985 & 257 & 465 & 211 & 450 \\
\hline 1986 & 300 & 457 & 223 & 456 \\
\hline 1987 & 363 & 450 & 194 & 452 \\
\hline 1988 & 522 & 443 & 310 & 444 \\
\hline 1989 & 532 & 439 & 359 & 431 \\
\hline 1990 & 461 & 424 & 456 & 421 \\
\hline 1991 & 544 & 418 & 413 & 416 \\
\hline 1992 & 343 & 405 & 309 & 401 \\
\hline 1993 & 216 & 375 & 103 & 392 \\
\hline
\end{tabular}


1034

Tabela 8 - Componentes de variância genética aditiva $\left(\sigma_{a}{ }^{2}\right)$, ambiental $\left(\sigma_{e}^{2}\right)$, fenotípica $\left(\sigma_{p}^{2}\right)$, herdabilidade $\left(h^{2}\right)$ e proporção do componente ambiental em relação ao fenotípico total $\left(1-h^{2}\right)$, para peso adulto

Table 8 - Genetic additive $\left(\sigma_{a}^{2}\right)$, environmental $\left(\sigma_{e}^{2}\right)$ and phenotypic $\left(\sigma_{p}^{2}\right)$ variance components, heritability $\left(h^{2}\right)$ and proportion of environmental in relation to total phenotypic component $\left(1-h^{2}\right)$ for mature weight

\begin{tabular}{ccccc}
\hline$\sigma_{\mathrm{a}}{ }^{2}$ & $\sigma_{\mathrm{e}}{ }^{2}$ & $\sigma_{\mathrm{p}}{ }^{2}$ & $\mathrm{~h}^{2}$ & $1-\mathrm{h}^{2}$ \\
\hline 537,78 & $1.553,22$ & $2.090,59$ & 0,26 & 0,74 \\
\hline
\end{tabular}

$\left(1.553,22 \mathrm{~kg}^{2}\right)$ e fenotípico $\left(2.090,59 \mathrm{~kg}^{2}\right)$ que constam da Tabela 8 .

A estimativa da herdabilidade do peso adulto encontrada $(0,26)$ nesta pesquisa situou-se abaixo da média dos valores reportados na literatura, para raças de origem européia (0,50; KOOTS et al., 1994). Para o caso de dados da raça Nelore no Brasil, no entanto, foram encontradas na literatura referências de apenas quatro estimativas, três delas para pesos tomados na assíntota da curva de crescimento (DUARTE, 1975: 0,69; LUDWIG et al., 1979: 0,28; CARRIJO, 1988: 0,09 ) e apenas uma, no valor de 0,34 , para dados de campo (ROSA et al., 2000).

Embora KOOTS et al. (1994) não tenham observado diferenças nas estimativas devidas à origem dos dados (campo ou experimentais) e mesmo devidas à metodologia utilizada (REML ou Quadrados Mínimos), a diferença da estimativa aqui encontrada em relação às demais pode ser creditada, principalmente, à fonte de dados, no que se refere a amostragem. No caso da raça Nelore no Brasil, todas as quatro estimativas de herdabilidade disponíveis são provenientes de informações de um único rebanho. Nestas condições, com variações ambientais menores, reduz-se também o componente da variância fenotípica de modo que a proporção da variância genética aditiva, em relação à total, se eleva.

A estimativa de herdabilidade encontrada no presente trabalho, de média magnitude, pode ser considerada de relevante valor prático uma vez que o peso adulto não estaria sendo sujeito a aumentos acentuados, como resposta à seleção, preocupação marcante para a maioria das raças bovinas de corte, por suas implicações negativas sobre a eficiência reprodutiva e econômica, em geral.

Os valores genéticos preditos para todos os animais

\section{OSA et al.}

integrantes da matriz de parentesco variaram entre $-41,9$ e $+61,4$ kg, com acurácias entre 0,0 e 0,95, sendo o coeficiente de consangüinidade médio estimado na população, praticamente, nulo (Tabela 9).

Embora a amplitude de variação destes valores genéticos seja bastante elevada, da ordem de $102 \mathrm{~kg}$, observou-se, no entanto, que a frequiência acumulada entre os valores genéticos, de $-18 \mathrm{a}+18 \mathrm{~kg}$, representou mais de $90 \%$ da distribuição (Figura 1).

A predição dos valores genéticos de touros com oito ou mais filhas, em número de 302 para toda a população, demonstrou a viabilidade de inclusão do peso adulto nos sumários de avaliação genética, como suporte à tomada

Tabela 9 - Número de animais (N), média, desvio-padrão (DP) e valores mínimo e máximo para coeficiente de consangüinidade $(F)$, valor genético predito para o peso adulto (VGP) e acurácia

Table 9 - Number of animals (N), mean, standard deviation (SD) and minimum and maximum values for inbreeding coefficient $(F)$, predicted genetic breeding value for mature weight (PBV) and accuracy

\begin{tabular}{lcllcc}
\hline $\begin{array}{l}\text { Variável } \\
\text { Variable }\end{array}$ & $\mathrm{N}$ & $\begin{array}{c}\text { Média } \\
\text { Mean }\end{array}$ & $\begin{array}{l}\mathrm{DP} \\
\text { SD }\end{array}$ & $\begin{array}{c}\text { Mínimo } \\
\text { Minimum }\end{array}$ & $\begin{array}{c}\text { Máximo } \\
\text { Maximum }\end{array}$ \\
\hline $\mathrm{F}$ & 17.039 & 0,006 & 0,024 & 0,0 & 0,380 \\
VGP $(\mathrm{kg})$ & 17.039 & 1,7 & 9,5 & $-41,9$ & 61,4 \\
$P B V(\mathrm{~kg})$ & & & & & \\
Acurácia & 17.039 & 0,39 & 0,20 & 0,0 & 0,95 \\
Accuracy & & & & & \\
\hline
\end{tabular}

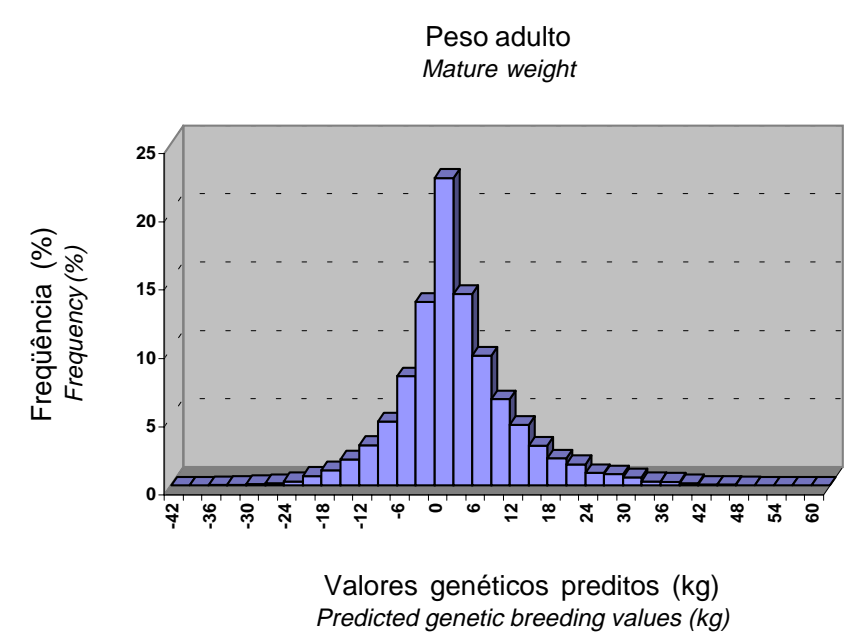

Figura 1 - Distribuição de freqüência dos valores genéticos preditos para peso adulto $(\mathrm{kg})$.

Figure 1 - Mature weight predicted genetic breeding values $(\mathrm{kg})$ frequency distribution. 
Rev. bras. zootec.

Tabela 10 - Valor genético predito para o peso adulto (VGP), acurácia, número de filhas (N) e relação dos reprodutores por nome e registro genealógico definitivo (RGD) 1

Table 10 - Predicted genetic breeding value (PBV), accuracy, number of daughters $(N)$ and sire summary by name and genealogical registration number $(G R N)^{1}$

\begin{tabular}{|c|c|c|c|c|c|}
\hline $\begin{array}{l}\text { RGD } \\
G R N\end{array}$ & $\begin{array}{l}\text { Nome } \\
\text { Name }\end{array}$ & $\begin{array}{l}\text { VGP }(\mathrm{kg}) \\
P B V(k g)\end{array}$ & $\begin{array}{l}\text { Acuracia } \\
\text { Accuracy }\end{array}$ & $\begin{array}{l}\mathrm{N} \\
N\end{array}$ & $\begin{array}{c}\text { Quartil } \\
\text { Quartile }\end{array}$ \\
\hline C3669 & ISAKHAN N. INDIA & $-35,8$ & 0,60 & 11 & 1 \\
\hline C6869 & GALECIODA STA FIL & $-33,6$ & 0,84 & 35 & 1 \\
\hline D 8268 & BINGO & $-31,2$ & 0,65 & 10 & 1 \\
\hline C4995 & SENARIODAZEB. VR & $-3,0$ & 0,58 & 9 & 1 \\
\hline C 8926 & JHANSI POI DA BV & $-3,0$ & 0,55 & 8 & 1 \\
\hline 3387 & EVEREST III - 15 & $-2,8$ & 0,68 & 28 & 1 \\
\hline H 1753 & PALERMO & $-2,4$ & 0,33 & 8 & 2 \\
\hline $\mathrm{C} 0522$ & FALLONDOSABIA & $-2,2$ & 0,83 & 40 & 2 \\
\hline В 0032 & UFANGI-POI & $-2,0$ & 0,61 & 10 & 2 \\
\hline A 5250 & LABANDAZEB & 0,4 & 0,67 & 16 & 2 \\
\hline B 1922 & BACURI & 0,4 & 0,39 & 9 & 2 \\
\hline H4198 & MASCATE DA NI & 0,4 & 0,59 & 10 & 2 \\
\hline C5476 & RHUBAYA POIZEB- & 0,6 & 0,67 & 15 & 3 \\
\hline H7542 & RIACHO & 0,6 & 0,90 & 129 & 3 \\
\hline 7244 & DAGHA & 0,8 & 0,48 & 8 & 3 \\
\hline 3174 & PALACIO & 4,2 & 0,26 & 9 & 3 \\
\hline $\mathrm{C} 0280$ & CALCUTA POIDOBR & 4,2 & 0,79 & 62 & 3 \\
\hline $\mathrm{C} 0318$ & KAMUK & 4,4 & 0,63 & 16 & 3 \\
\hline H 1076 & FALODABV & 4,6 & 0,62 & 13 & 4 \\
\hline A 1753 & GADY DA SC & 4,8 & 0,68 & 19 & 4 \\
\hline A 1514 & GANAPATHY DOBR & 4,8 & 0,22 & 8 & 4 \\
\hline D 0223 & LEVANTEDAFAZ. & 47,0 & 0,75 & 15 & 4 \\
\hline C6740 & LUDY DEGARCA & 50,6 & 0,95 & 212 & 4 \\
\hline F9902 & ZEFEC ABDALA & 52,2 & 0,76 & 33 & 4 \\
\hline
\end{tabular}

${ }^{1}$ Inclui apenas três touros em cada um dos extremos dos quartis da distribuição; a avaliação completa incluiu 302 touros com número de filhas maior ou igual a oito.

1 It includes only three sires in each one of the quartiles extremes of the distribution; the whole relation had 302 sires that had at least eight progenies evaluated.

de decisões de criadores e de técnicos, na execução dos programas de seleção (Tabela 10).

Embora o valor de herdabilidade tenha sido de média magnitude, a amplitude dos valores genéticos preditos aqui encontrada comprova que a raça Nelore apresenta recursos genéticos suficientes para o suporte de programas de seleção na busca por biótipos de diferentes tamanhos, confirmando as expectativas de levantamentos como os feitos por MAGNABOSCO et al. (1997), com relação às diferentes linhagens fundadoras desta raça, no Brasil.

\section{Conclusões}

A média geral estimada por quadrados mínimos, $447 \mathrm{~kg}$, e o desvio médio para peso adulto, $50 \mathrm{~kg}$, permitem caracterizar a raça Nelore como uma raça de porte médio. A estimativa da herdabilidade, de média magnitude, 0,26, indica ser esta característica passível de alterações por meio de seleção dentro ou entre rebanhos. Os valores genéticos preditos de peso adulto na população analisada, variando entre $-41,9 \mathrm{e}+61,4 \mathrm{~kg}$, indicam a existência de recursos genéticos que, aliados às intensidades de seleção adequadas, a cada caso, poderão contribuir para o atendimento de diferentes demandas dos sistemas de produção, conforme as condições ambientais e os recursos disponíveis, em termos de animais de pequeno, médio e grande porte.

\section{Agradecimento}

À Embrapa-Empresa Brasileira de Pesquisa Agropecuária, pela oportunidade do curso; à CAPES, pela valiosíssima bolsa de pós-graduação; ao CNPq/ RAHE e à FINEP/BID, pelo suporte financeiro do programa; aos criadores, pela coleta e disponibilidade das informações; aos técnicos do Programa de Melhoramento Genético da Raça Nelore, pelo suporte no gerenciamento da base de dados; ao Pesquisador Dr. Luiz Otávio Campos da Silva, conselheiro acadêmico do autor, pelo suporte durante o curso e, especialmente, na fase de editoração do trabalho de tese. 


\section{Referências Bibliográficas}

ANUALPEC 98. São Paulo: FNP, 1998. 385p.

ARRUDA, Z.J., SUGAI, Y. 1994. Regionalização da pecuária bovina no Brasil. Campo Grande: EMBRAPA-CNPGC. 144p. (Documentos, 18).

BOLDMAN, K.G., KRIESE, L.A., VAN VLECK, L.D. et al. 1993. A manual for use of MTDFREML- a set of programs to obtain estimates of variances and covariances (DRAFT). Lincoln: USDA-ARS. 114p.

CARRIJO, S.M. Descrição e comparação de parâmetros de crescimento de animais das raças Chianina e Nelore. Ribeirão Preto, SP: USP, 1988. 116p. Dissertação (Mestrado) Faculdade de Medicina de Ribeirão Preto, Universidade de São Paulo, 1988.

CORTARELLI, A. Estudo da curva de crescimento de machos da raça Nelore através de quatro modelos estocáticos. Jaboticabal, SP: UNESP, 1973. 179p. Tese (Doutorado) Faculdade de Medicina Veterinária e Agronomia de Jaboticabal, Universidade Estadual Paulista, 1973.

DUARTE, F.A.M. Estudo da curva de crescimento de animais da raça "Nelore" (Bos taurus indicus) através de cinco modelos estocásticos. Ribeirão Preto, SP: USP, 1975. 284p. Tese (Livre Docência) - Faculdade de Medicina de Ribeirão Preto, Universidade de São Paulo, 1975.

ELIAS, A.M., PACKER, I.U., RAZOOK, A.G. et al. Peso assintótico e taxa de maturidade de vacas das raças Nelore, Guzerá e Gir. In: REUNIÃO ANUAL DA SOCIEDADE BRASILEIRA DEZOOTECNIA, 35, 1998, Botucatu. Anais... Botucatu: SBZ, 1998, v. 3., p.324-326.

ENSMINGER, M.E. 1987. Beef cattle science. Danville: The Interstate. $1030 \mathrm{p}$.

FELIUS, M. 1985. Genus Bos: cattle breeds of the world. New York: Merk, 234p.

FITZHUGH, H.A. 1976. Analysis of growth curves and strategies for altering their shape. J. Anim. Sci., 42(4):1036-1051.

HOHENBOKEN, W.D. Genetic x environment interactions and animal production: when nurture and nature collide. In: REUNIÃO DA SOCIEDADE BRASILEIRA DE ZOOTECNIA, 33, 1996, Fortaleza. Simpósio... Fortaleza: SBZ, 1996. p.21-24.

HOHENBOKEN, W.D. Physiological limits to selection in beef and dairy cattle: evidence and expectations from diverse sources. In: REUNIÃO DA SOCIEDADE BRASILEIRA DEZOOTECNIA, 35, 1998, Botucatu. Simpósio... Botucatu: SBZ, 1998. p.371-397.

JENKINS, T.G., FERRELL, C.L. 1994. Productivity through weaning of nine breeds of cattle under varying feed availabilities. I. Initial evaluation. J. Anim. Sci., 72(11):2787-2797.

KOOTS, K.R., GIBSON, J.P., SMITH, C. et al. 1994. Analyses of published genetic parameters estimates for beef production traits. I. Heritability. Anim. Breed. Abstr., 62(5):309-338.
LANNA, D.P., PACKER, I.U. Eficiência biológica e econômica de bovinos de corte. In: QUALIDADE DA CARNE E MELHORAMENTO GENÉTICO DE BOVINOS DE CORTE. Anais... São Carlos: Embrapa-CPPSE-FUNDEPEC, 1998. p. 83-104. versão preliminar.

LÔBO, R.B. 1996. Programa de melhoramento genético da raça Nelore. Ribeirão Preto, SP: PMGRN. 104p.

LUDWIG, A., SILVA, M.A., GOMES, F.R. 1979. Análise genética dos parâmetros de curvas de crescimento de gado nelore. R. Bras. Zootec., 8(1):133-151.

MAGNABOSCO, C.U., CORDEIRO, C.M.T., TROVO, J.B.D.F. et al. 1997. Catálogo de linhagens do germoplasma zebuíno: raça Nelore. Brasília: Embrapa-Cenargen. 52p. (Documentos, 23).

NOBRE, P.R.C., ROSA, A.N., EUCLIDES FILHO, K. 1987a. Interação genótipo $\mathrm{x}$ ambiente em gado Nelore. R. Bras. Zootec., 16(4):352-363.

NOBRE, P.R.C., ROSA, A.N., SILVA, L.O.C. et al. 1987b. Curvas de crescimento de gado Nelore ajustadas para diferentes frequiências de pesagens. Pesq. Agropec. Bras., 22(9/10):1027-1037.

PETERS, R.H., 1993. The ecological implications of body size. New York: Cambridge University Press. 329p.

RITCHIE, H.D. The optimum cow: what criteria must she meet? In: BEEF IMPROVEMENT FEDERATION ANNUAL CONFERENCE, 1995, Sheridan. Proceedings... Sheridan: Beef Improvement Federation. p.126-145.

ROSA, A.N., SILVA, M.A., LUDWIG, A. 1978. Parâmetros genéticos e fenotípicos de pesos corporais ajustados pela curva de crescimento de animais da raça Nelore. R. Bras. Zootec., 7(2):329-345.

ROSA, A.N., LÔBO, R.B., OLIVEIRA, H.N. et al. 2000. Variabilidade genética do peso adulto de matrizes em um rebanho Nelore no Estado de São Paulo. Rev. bras. zootec., 29(6):1690-1695.

SAS INSTITUTE, 1995. SAS user's guide for windows environment. 6.08 ed. Cary: SAS Institute Inc. 956p.

SILVA, L.O.C. Tendência genética e interação genótipo x ambiente em rebanhos Nelore criados a pasto no Brasil Central. Viçosa: UFV, 1990. 113p. Tese (Doutorado) - Escola Superior de Agronomia, Universidade Federal de Viçosa, 1990.

Recebido em: 09/08/00

Aceito em: 09/01/01 